\title{
Emmentaler Gesundheitskostenstatistik
}

\section{Ärztenetzwerk \\ oberes Emmental}

Interessenverbindungen und mögliche Interessenkonflikte: Die Erhebung der Daten bei santésuisse wurde aus privaten Spenden finanziert. Die Arbeit der Autoren war unentgeltlich.
Korrespondenz:

Dr. med. Markus Bieri-Zürcher

Facharzt für Allgemeinmedizin FMH Lindenstrasse 7

CH-3550 Langnau im Emmental
1999 fusionierten das Regionalspital Burgdorf (unteres Emmental) und das Bezirksspital Langnau (oberes Emmental) zur Regionalspital Emmental AG. Aus betriebswirtschaftlichen Gründen wurde im Jahr 2003 die Schliessung der stationären Chirurgie in Langnau in Erwägung gezogen. Die lokale Ärzteschaft befürchtete eine Schliessung des Akutbetriebes in Raten. Im Rahmen einer breiten Oppositionsbewegung wurde im Sommer 2004 eine Petition mit 37000 Unterschriften Regierungsrat Samuel Bhend übergeben. Beigelegt war ein Argumentarium für den Erhalt des lokalen Akutspitals. Besonders gewichtet wurden volkswirtschaftliche Aspekte. In der Folge entstand das Bedürfnis, diese Sichtweise mit Zahlen zu untermauern.

\section{Ausgangslage}

Das obere Emmental ist eine weitläufige, stark ländliche Region mit 25000 Einwohnern. Es fällt in der Bevölkerung ein proportional höherer Anteil an älteren Menschen auf, was auf den Niedergang der Landwirtschaft und den Mangel an Arbeitsplätzen zurückzuführen ist. Beispielsweise beträgt der Anteil der über 65jährigen schweizweit 15,02\%, im oberen Emmental sind es 17,06\%. Medizinisch sind ambulant 19 Grundversorger, 2 Gynäkologen, 2 Psychiater und 1 Augenarzt tätig. Im lokalen 70-Betten-Spital, das noch grosse Teile der angrenzenden Regionen versorgt, arbeiten 3 Internisten und 3 Chirurgen in leitender Funktion. Sie decken auch ein breites Spektrum an technischen Untersuchungen ab. Die Bindung zum Hausarzt ist überdurchschnittlich, der direkte Gang zum Spezialisten die Ausnahme. Zudem wurde 1998 im Spital eine komplementärmedizinische Station eröffnet, die aktuell 12 Betten umfasst und von 3 auch ambulant in der Region tätigen Kollegen geleitet wird. Was die Krankenkassenkosten anbelangt, so zählt das obere Emmental zur billigsten Prämienregion innerhalb des Kantons.

\section{Ziel der Studie}

Es sollte aufgrund der Kosten pro Versicherten statistisch erfasst werden, um wieviel und in welchen Bereichen sich die volkswirtschaftlichen Gesundheitskosten des oberen Emmentals von

\section{Statistique emmentaloise \\ des coûts de la santé}

Une analyse rétrospective de santésuisse compare les coûts par assuré en Suisse, dans le canton de Berne et le Haut-Emmental au cours des années 2001 à 2005. Dans le domaine ambulatoire, les coûts des traitements de spécialistes en Emmental sont la moitié moins élevés, probablement en raison de la fonction des traditionnels médecins de premiers recours (gatekeeper). Les dépenses nettement plus basses par assuré pour les soins hospitaliers sont dus à l'hôpital régional, qui fournit une assistance de base avantageuse et effectue par la même occasion un tri des patients efficace sur le plan des coûts. Le concept-modèle médecin de famille et hôpital de premier recours s'avère efficace.

jenen anderer Regionen unterscheiden. Zudem sollte geklärt werden, ob sich daraus Schlüsse für die optimale Versorgung dieser Region ziehen lassen, die allenfalls verallgemeinert werden dürfen.

\section{Methode}

Es war nicht möglich, eigens eine prospektive Studie mit genügend verlässlichen Daten durchzuführen. Deshalb wurde beschlossen, auf die detaillierten Zahlen der santésuisse-Statistik der Jahre 2001-2005 zurückzugreifen, worin 97\% aller von den Krankenkassen bezahlten Kosten erfasst sind. Entscheidend waren die Kosten pro Versicherten, und zwar 1. der gesamten Schweiz, 2. des Kantons Bern und 3. des oberen Emmentals, unabhängig davon, wo sie verursacht wurden. Beispielsweise wurden also auch die Spitalkosten erfasst, die die Emmentaler nicht in Langnau verursachten. Das Zahlenmaterial der santésuisse ist verlässlich und objektiv; ein möglicher «selection bias» entfällt. Die Daten 
wurden einer deskriptiven, retrospektiven Analyse unterzogen.

\section{Resultate}

Abbildung 1 zeigt, was hinlänglich bekannt ist: Die Kosten pro Versicherten für die Entschädigung der Grundversorger stieg in den letzten fünf Jahren nur geringfügig - nämlich schweizweit um 4,2\%; eine Konstanz, die auch im Rahmen der TARMED-Einführung verlangt wurde. Auffällig ist die geringe Differenz der Zahlen für die Schweiz, den Kanton Bern und das obere Emmental.

Grosse Unterschiede zeigen sich bei den Kosten pro Versicherten für Spezialärzte in der Abbildung 2. Schweizweit und im Kanton Bern werden dafür gut Fr. 400.- pro Versicherten ausgegeben, im oberen Emmental nur Fr. 208.-. Bemerkenswert ist, dass schweizweit die Kosten pro
Versicherten für Spezialärzte $183 \%$ jener für Grundversorger ausmachen, während dies im oberen Emmental nur gerade 93\% sind. Dieses Verhältnis hat sich im Verlauf der letzten fünf Jahre nicht verändert.

Abbildung 3 zeigt den Verlauf der Kosten pro Versicherten für stationäre Spitalbehandlungen. Diese sind schweizweit zwischen 2001 und 2005 um $27 \%$ angestiegen. Im Kanton Bern fällt eine vergleichsweise grosse Zunahme von $83 \%$ auf. Im Emmental sind die Spitalkosten pro Versicherten um 75\% gestiegen, sie sind aber 2005 immer noch $23 \%$ niedriger als im Durchschnitt des Kantons Bern.

In der Abbildung 4 werden die gesamten Medikamentenkosten pro Versicherten dargestellt. Die Unterschiede in den drei Gruppen sind relativ gering, das obere Emmental liegt 11\% unter dem schweizerischen Durchschnitt.

Abbildung 1

Kosten pro Versicherten für Grundversorger.

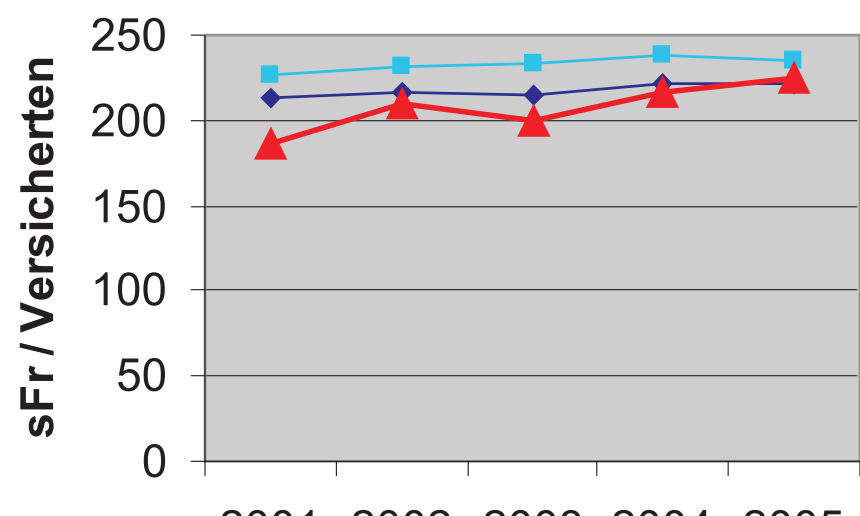

$\multimap \mathrm{CH}$ gesamt

20012002200320042005

Abbildung 2

Kosten pro Versicherten für Spezialärzte.

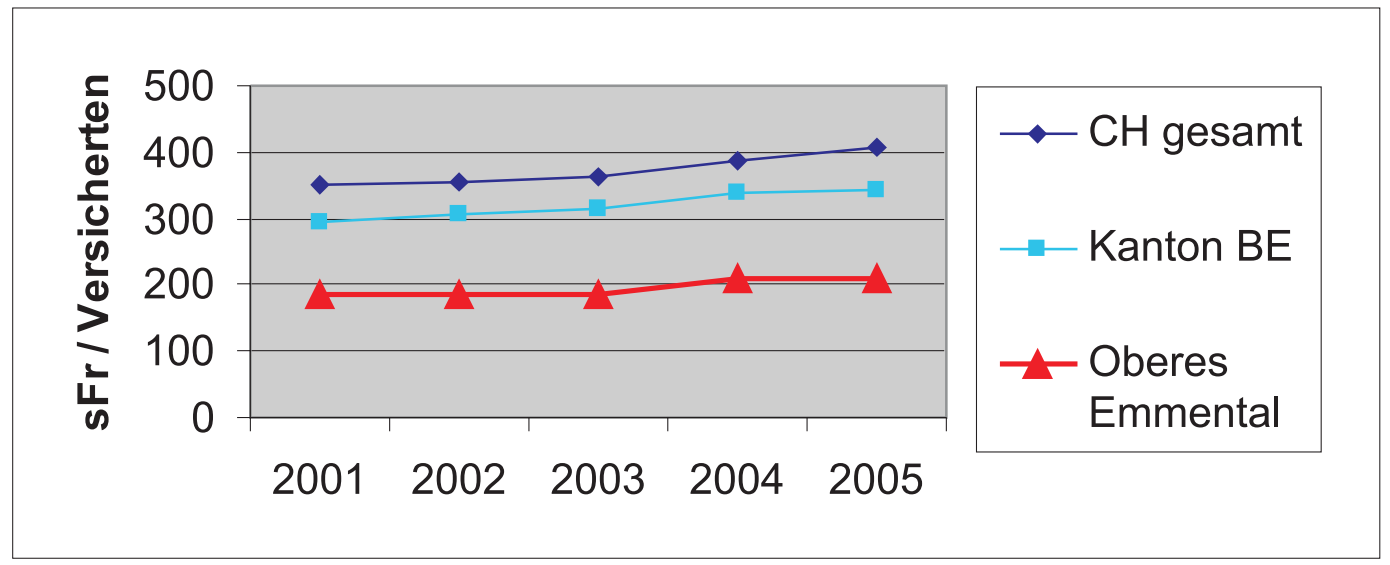




\section{Kritik}

Studien im Gesundheitswesen sind mit dem Problem behaftet, dass praktisch nie Gleiches mit Gleichem verglichen wird. Das wird besonders augenfällig, wenn die Kosten von Spitälern einander gegenübergestellt werden, hat doch fast jedes Krankenhaus einen unterschiedlichen Auftrag und unterschiedliche Schwerpunkte. In der vorliegenden Studie wurden nicht Institutionen oder Fälle miteinander verglichen, sondern die Kosten pro Versicherten einander gegenübergestellt.

Bei einer Bevölkerung von 25000 Einwohnern im oberen Emmental darf davon ausgegangen werden, dass sich die Krankheitshäufigkeit in dieser Region nicht wesentlich von anderen Gegenden unterscheidet. Wenn es in urbanen
Regionen beispielsweise mehr Krankheiten infolge des Drogenabusus gibt, sind in ländlichen Regionen degenerativ-rheumatische Erkrankungen mit entsprechenden Folgekosten im Bereich der Orthopädie höher.

Der Einwand, dass die Landbevölkerung weniger häufig ärztliche Hilfe in Anspruch nimmt, mag teilweise die eklatant tieferen Kosten für Spezialärzte erklären. Hingegen wird sich die geringere Anspruchshaltung kaum auf die stationäre Behandlungsbedürftigkeit auswirken. Gerade der hohe Anteil älterer Menschen im oberen Emmental legt nahe, dass dort eher häufiger Hospitalisationen notwendig werden.

Die Tatsache, dass die Medikamentenkosten pro Versicherten nur gering unter dem schweizerischen Durchschnitt liegen, lässt den Schluss

Abbildung 3

Kosten pro Versicherten für stationäre Spitalbehandlungen.

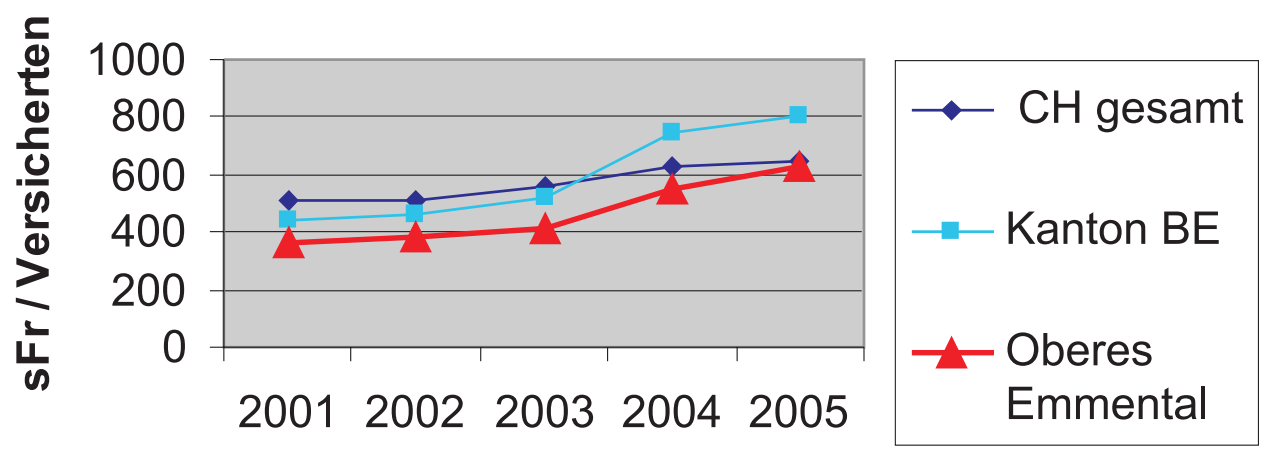

Abbildung 4

Kosten pro Versicherten für Medikamente.

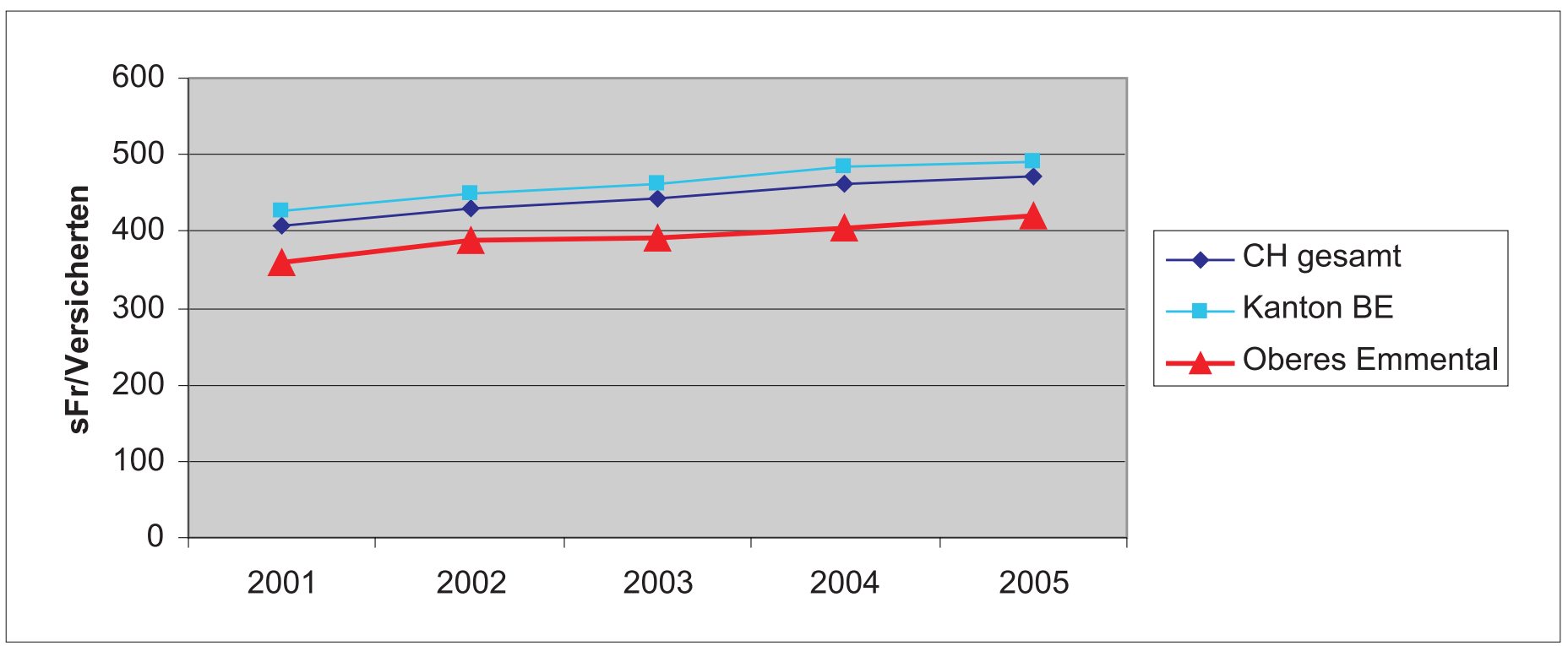


zu, dass die Menschen im oberen Emmental medizinisch nicht weniger intensiv betreut werden.

Dem Einwand, dass staatliches Geld via öffentliche Spitalfinanzierung vor allem in ländliche Regionen fliesst und damit die lokalen Krankenkassenkosten tiefer hält, muss entgegengehalten werden, dass ausser der Stadt und Agglomeration Bern das Emmental innerhalb des Kantons die kleinsten öffentlichen Pro-KopfBeiträge für die Spitäler erhält.

\section{Schlussfolgerung}

Unter Würdigung der genannten Einwände lassen die erhobenen Zahlen folgende Schlüsse $\mathrm{zu}$ :

1. Der wesentliche Unterschied bezüglich ambulanter Kosten zwischen dem oberen Emmental und dem Durchschnitt des Kantons Bern bzw. der Schweiz liegt beim Aufwand für Spezialärzte. Da wirkt sich aus, dass einerseits Spezialisten vor Ort fehlen und andererseits de facto ein Hausarztmodell mit Gatekeeping schon immer bestanden hat.

2. Das Augenfälligste bei der Analyse der stationären Spitalkosten ist der überdurchschnittliche Anstieg im Kanton Bern, wobei er im oberen Emmental nur unwesentlich geringer ist. Diese Entwicklung dürfte wesentlich auf gesundheitspolitische Entscheide zurückzuführen sein, indem im Kanton Bern neu allgemeinversicherte Patienten ohne Vorbehalt Zugang zu den staatlich nichtsubventionierten Privatspitälern erhalten haben.
3. Die Kosten pro Versicherten für stationäre Spitalbehandlungen im oberen Emmental liegen konstant $20 \%$ und mehr unter jenen des Kantons Bern. Die zentrale Frage, weshalb dieser Unterschied bestehen bleibt, kann unseres Erachtens nur durch einen Faktor erklärt werden: das System der stationären Gesundheitsversorgung an sich: Während in urbanen Regionen die Patienten primär einem grösseren Spital mit entsprechender Spezialisierung zugewiesen werden, erfolgen Spitaleinweisungen in ländlichen Gegenden in der Regel ins lokale Spital. Mindestens drei Viertel der Patienten können mit den vorhandenen, relativ bescheidenen Mitteln ausreichend behandelt werden, und nur ein kleiner Teil wird im Sinne einer Triage sekundär an ein spezialisiertes Zentrum überwiesen. Damit existiert in ländlichen Gegenden wie im oberen Emmental nicht nur ein Hausarztmodell im ambulanten Bereich, sondern auch im stationären Bereich ein volkswirtschaftlich relevantes «Hausspitalmodell». Aufgrund der vorliegenden Zahlen kann sogar gefragt werden, ob gesundheitspolitisch ein Modell mit einerseits dezentralen Spitälern mit reinem Grundversorgungsauftrag und andererseits wenigen spezialisierten Zentrumsspitälern nicht effizienter, kostengünstiger und patientengerechter wäre. Vielleicht liesse sich auch auf diesem Wege eine letztlich unumgängliche Rationierung in der stationären Medizin menschlich vertretbar einführen. 